International Mathematical Forum, 2, 2007, no. 14, 699 - 704

\title{
Some results to the Huzita axioms
}

\author{
H. R. Khademzadeh and H. Mazaheri \\ Department of Mathematics \\ Yazd University, Yazd, Iran \\ hmazaheri@yazduni.ac.ir
}

\begin{abstract}
Origami is a Japanese traditional art of paper folding. The word origami is a combined word coming from ori (fold) and kami (paper). Huzita axioms are useful principle in the origami. In the Huzita axioms think about this question, when there is an unique fold in the five axioms? or in the six axiom? In this paper we want to answers to these questions.
\end{abstract}

\section{Mathematics Subject Classification: 68T15}

Keywords: Origami, Huzita axioms, Fold, Line fold.

\section{Introduction}

For several centuries, origami has been popular among Japanese common people as an art, as a playing toy, and teaching material for children. Over a decade, origami is also receiving wide interest among mathematicians, mathematics educators and computer scientists, as well as origami artists, as origami poses interesting fundamental geometrical questions.

An origami is to be folded along a specified line on the origami called fold line. The line segment of a fold line on the origami is called a crease, since the consecutive operations of a fold and an unfold along the same fold line makes a crease on the origami. A fold line can be determined by the points it passes through or by the points (and/or lines) it brings together. As in Euclidean geometry, by specifying points, lines and their configuration, we have the following six basic fold operations called origami axioms of Huzita [Huzita 1989, Hull 1997]. It is known that Huzita's origami axiom set is more powerful than the ruler-and-compass method in Euclidean geometry [Geretschlaeger 2002]. Origami can construct objects that are impossible by the ruler-andcompass method [Chen 1966]. One of them is trisecting an angle. 


\section{Origami Axioms}

Huzita's origami axioms are described in term of fold operations as follows:

(O1) Given two points, we can make a fold along the crease passing through them.

(O2) Given two points, we can make a fold to bring one of the points onto the other.

(O3) Given two lines, we can make a fold to superpose the two lines.

(O4) Given a point $\mathrm{P}$ and a line $\mathrm{m}$, we can make a fold along the crease that is perpendicular to $\mathrm{m}$ and passing through $\mathrm{P}$.

(O5) Given two points $\mathrm{P}$ and $\mathrm{Q}$ and a line $\mathrm{m}$, either we can make a fold along the crease that passes through $\mathrm{Q}$, such that the fold superposes $\mathrm{P}$ and $\mathrm{m}$, or we can determine that the fold is impossible.

(O6) Given two points $\mathrm{P}$ and $\mathrm{Q}$ and two lines $\mathrm{m}$ and $\mathrm{n}$, either we can make a fold along the crease, such that the fold superposes $\mathrm{P}$ and $\mathrm{m}$, and $\mathrm{Q}$ and $\mathrm{n}$, simultaneously, or we can determine that the fold is impossible.

In axiom $O_{5}$ we can show that, if $|P Q|=d(Q, m)$ then there is an unique fold. Where:

$$
d(Q, m)=\inf _{R \in m}|Q-R|
$$

\section{Main Results}

We see in some axioms that two or three folds exist such that satisfied in axiom. For example in $O_{6}$ we know that it is equivalent to solving a cubic equation and therefore sometimes there are one, two or three folds that satisfied in the $O_{6}$. But for example in the $O_{1}$ there is an unique fold. Also for $O_{2}$ and $O_{4}$.

Question: When there is an unique fold in the five axiom?

We can answer to this question with three theorems.

\section{Definitions:}

a) For the points $P$ and $Q$ and the line $m$, if there is an unique fold satisfy in 
$O_{5}(P, Q, m)$ then we denoted this fold by $[P, Q, m]$.

b) For the points $P$ and $Q$ and the lines $n$ and $m$, if there is an unique fold in $O_{6}(P, Q, m, n)$, then we denoted this fold by $[P, Q, m, n]$.

Theorem 1.2. The geometry locus of the points $Q$, that there exits the fold $[P, Q, m]$ is a parabola with the focus on $P$ and directrix $m$.

Proof: Suppose that the point $P\left(x_{1}, y_{1}\right)$ and the line $m: y=a x+b$ be given and $Q(x, y)$ be an arbitrary point in $R^{2}$. It is known that if $|P Q|=$ $d(Q, m)$ then there is an unique fold such that satisfied in the $O_{5}$. The above equation is a a parabola equation so the geometry locus of the point $Q$ is a parabola with focus at the $P$ and the directrix $m$.

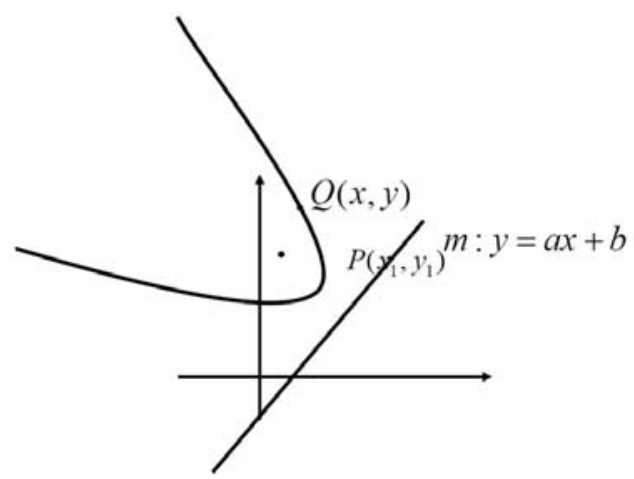

Figure 1

Theorem 2.2. The geometry locus of the point $P$ such that there exist a fold $[P, Q, m]$, is a circle with the center at $Q$ and radius $d(Q, m)$.

Proof: Suppose the point $Q\left(x_{2}, y_{2}\right)$ and the line $m: y=a x+b$ in the $O_{5}$ be given. If for some point $P(x, y)$ in $R^{2},[P, Q, m]$ be an unique fold in the $O_{5}$ then $|P Q|=d(Q, m)$ and we have:

$$
\left(x-x_{2}\right)^{2}+\left(y-y_{2}\right)^{2}=\frac{\left(y_{2}-a x_{2}-b\right)^{2}}{1+a^{2}}
$$

That is a circle equation. So the geometry locus of all point $P$ is a circle with center at $Q$ and radius $d(Q, m)$ it is obvious that for the point in the circle there isn't any fold and for the point out of the circle there are two folds. 


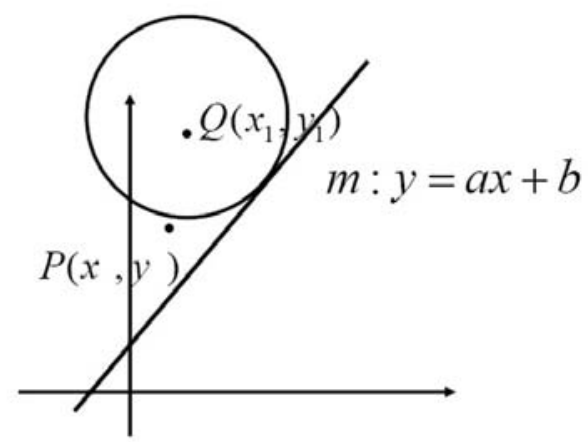

Figure 2

Theorem 3.2. The set of lines $m$, where $[P, Q, m]$ is a fold for some $P, Q$ is the set of all lines that is tangent to the circle $C$ with center at $Q$ and radius $|P Q|$.

Proof: From the preceding two theorems is clear.

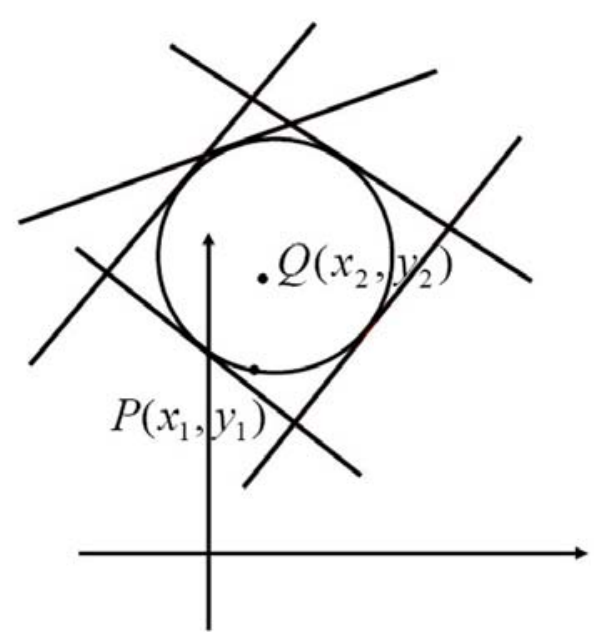

Figure 3

Now suppose in the $O_{6}$ the lines $m, n$ be parallel and we have the equations of $m, n$ as follows:

$$
m: y=a x+b_{1} \quad \text { and } \quad n: y=a x+b_{2}
$$


And given a point $P\left(x_{1}, y_{1}\right)$ :

Theorem 4.2. Let the point $P\left(x_{1}, y_{1}\right)$, and the line $m, n$, be given the geometry locus of all points $Q(x, y)$ with fold $[P, Q, m, n]$ is a circle with center at $P$, and radius $d(m, n)$.

Proof: We know that $O_{6}$ is equivalent to solving a cubic equation such that the root of this equation is the slope of the line fold. with gyration the $\mathrm{x}$-axis and $\mathrm{y}$-axis we consider the equation of the lines $m, n$ as follows:

$$
m: y=b_{1} \quad \text { and } \quad n: y=b_{2}
$$

And suppose that $Q(x, y)$ be an arbitrary point in $R^{2}$. If we compute the equation of this line fold, $[P, Q, m, n]$ we have[7]:

$$
\begin{aligned}
& y_{2}=-\frac{x_{2}}{a}+\frac{\left(-y_{1}-b_{1}\right) a^{2}-2 x_{1} a+\left(y_{1}+b_{1}\right)}{-2 a^{2}} \\
& y_{2}=-\frac{x_{2}}{a}+\frac{\left(-y_{2}-b_{2}\right) a^{2}-2 x_{2} a+\left(y_{2}+b_{2}\right)}{-2 a^{2}}
\end{aligned}
$$

And if we equal to other two these lines:

$$
\left(y-y_{1}+b_{2}-b_{1}\right) a^{2}+\left(2 x-2 x_{1}\right) a+\left(-y+y_{1}+b_{2}-b_{1}\right)=0
$$

That is a quadratic equation. and this equation have an unique root if the discriminant of equation equal to zero. So:

$$
\left(x-x_{1}\right)^{2}-\left(\left(b_{2}-b_{1}\right)+\left(y-y_{1}\right)\right)\left(\left(b_{2}-b_{1}\right)-\left(y-y_{1}\right)\right)=0
$$

Thus:

$$
\left(x-x_{1}\right)^{2}+\left(y-y_{1}\right)^{2}=\left(b_{2}-b_{1}\right)^{2}
$$

That is a circle equation with center at $P$, and radius $d(m, n)$. 


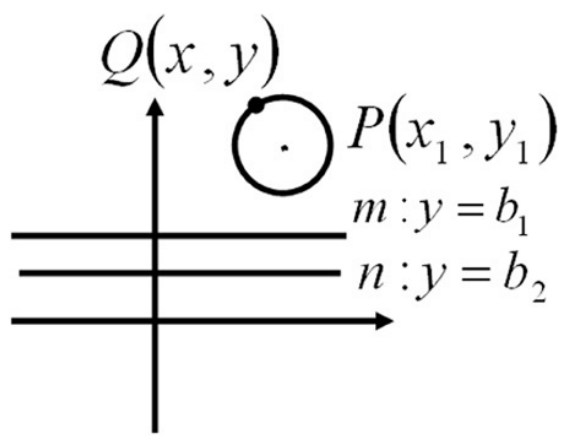

Figure 4

\section{References}

[1] R. Geretschlager, Geometric Constructions in Origami, Morikita Publising Co, 2002.

[2] T. L. Chen, Proof of the impossibility of trisecting an angle with Euclidean tools, Mathematics Magazine, 39 (1966), 239-241.

[3] T. Hull, On the mathematics of flat origami, Congressus Numerantium, 100 (1994), 215-224.

[4] H. Huzita, Axiomatic Development of Origami Geometry, Proceedings of the First International Meeting of Origami Science and Technology, (1989), $143-158$.

Received: January 27, 2006 